\title{
Spin-mediated Photomechanical Coupling of a Nanoelectromechanical Shuttle
}

\author{
A. V. Parafilo, ${ }^{1}$ S. I. Kulinich, ${ }^{2}$ L. Y. Gorelik, ${ }^{3}$ M. N. Kiselev, ${ }^{1}$ R. I. Shekhter, ${ }^{4}$ and M. Jonson ${ }^{4,5}$ \\ ${ }^{1}$ The Abdus Salam International Centre for Theoretical Physics, Strada Costiera 11, I-34151 Trieste, Italy \\ ${ }^{2}$ B. Verkin Institute for Low Temperature Physics and Engineering of the National Academy of Sciences of Ukraine, \\ Prospekt Nauky 47, Kharkov 61103, Ukraine \\ ${ }^{3}$ Department of Physics, Chalmers University of Technology, SE-412 96 Göteborg, Sweden \\ ${ }^{4}$ Department of Physics, University of Gothenburg, SE-412 96 Göteborg, Sweden \\ ${ }^{5}$ SUPA, Institute of Photonics and Quantum Sciences, Heriot-Watt University, Edinburgh EH14 4AS, Scotland, United Kingdom
}

(Received 26 February 2016; published 29 July 2016)

\begin{abstract}
We show that nanomechanical vibrations in a magnetic shuttle device can be strongly affected by external microwave irradiation through photo-assisted electronic spin-flip transitions. Mechanical consequences of these spin flips are due to a spin-dependent magnetic force, which may lead to a nanomechanical instability in the device. We derive a criterion for the instability to occur and analyze different regimes of nanomechanical oscillations. Possible experimental realizations of the spin-mediated photomechanical instability and detection of the device backaction are discussed.
\end{abstract}

DOI: 10.1103/PhysRevLett.117.057202

The electric charge of electrons injected by tunneling into a nanodevice provides a means for coupling mechanical deformations of the device to electronic degrees of freedom. Different scenarios of nanoelectromechanical (NEM) action, which provide a number of new nanodevice functionalities, are based on a coupling via either the injected electrical current or via charge accumulated in the device (see, e.g., the reviews [1-3]). The electronic spin, which is usually almost decoupled from the charge degrees of freedom in bulk nonmagnetic metals, might also contribute to the mechanics of nanometer-sized devices. Reasons for this can be the amplified spin-orbit interaction in low dimensional nonmagnetic conductors [4-6] or the exchange interaction induced in magnetic NEM structures $[7,8]$.

Microwave electromagnetic fields are in general not expected to affect the mechanical operation of a nanodevice very much. This is because of the considerable mismatch between the vibration frequency of a typical nanomechanical resonator $(\sim 100 \mathrm{MHz})$ and the frequency of an electromagnetic field in the microwave-far-infrared region (0.1-1 THz). In this Letter, however, we show theoretically that the electronic spin accumulated in a mechanical resonator can mediate a strong coupling between a highfrequency electromagnetic field and low-frequency mechanical vibrations in a magnetic NEM system such as the one sketched in Fig. 1(a). The device shown there comprises a single-wall carbon nanotube (CNT) resonator suspended between ferromagnetic source and drain electrodes and a magnetic gate. The magnetization of the two electrodes are assumed to be antiparallel, while the magnetization of the gate is taken to be antiparallel to that of the source.

The inhomogeneous magnetic field created by the tipshaped gate gives rise to a Zeeman splitting of the CNT electronic energy levels that varies with the deflection of the CNT [see Figs. 1(a) and 1(b)] and hence corresponds to a magnetic force that depends on the net spin accumulated in the CNT. This is the mechanism for how spin couples to the CNT vibrations. Turning to the mechanism for how spin couples to the microwave field, we assume that the CNT is connected to the electrodes by high-resistance tunnel barriers and that it is short enough $(\lesssim 1 \mu \mathrm{m})$ for the spatial quantization of energy levels to be resolved and that we are in the Coulomb blockade regime of transport at low temperatures $(\sim 1 \mathrm{~K})$. This means that we can treat the $\mathrm{CNT}$ as a quantum dot (QD) with a single electron energy (a)

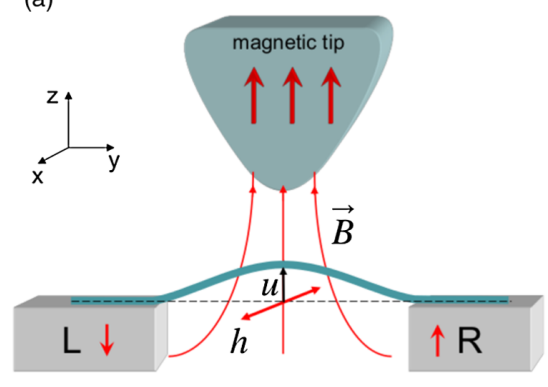

(b)

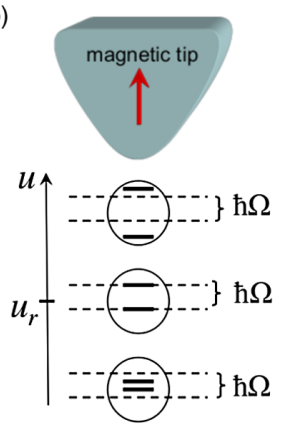

FIG. 1. (a) Sketch of a device with a suspended CNT resonator in tunneling contact with two ferromagnetic electrodes $(\mathrm{L}, \mathrm{R})$ with opposite magnetization directions. Flexural CNT vibrations are actuated by a magnetic force caused by the Zeeman interaction between the spin of CNT electrons and an inhomogeneous magnetic field $B_{z}(z)$ from a magnetic gate (tip). (b) A microwave field $h \cos (\Omega t)$ induces electron spin flips in the CNT, which are resonant at $u=u_{r}$ where the photon energy $\hbar \Omega$ equals the deflection-dependent Zeeman energy splitting $g \mu_{B} B_{z}(u)$. Since the magnetic force is spin dependent, the spin in this way mediates the photomechanical coupling discussed in the text. 
level, doubly degenerate due to the spin degree of freedom at zero magnetic field but Zeeman split at finite fields. The coupling of the microwave field to the electronic spin is through photo-assisted spin-flip scattering, which becomes resonant if the microwave photon energy equals the Zeeman splitting [see Fig. 1(b)]. Each spin flip changes the amount of spin accumulated in the CNT-QD in the sense that the value of the spin projection on the axis defined by the colinear magnetizations of electrodes and gate is changed by one unit, effectively adding an "extra spin" to the CNT resonator. Such photo-induced "spin pumping" also changes the spin-dependent magnetic force on the CNT. This, in qualitative terms, is how the electron spin can mediate an interaction between nanomechanical vibrations and a microwave field. To see what consequences such a coupling may have, we will ask the question whether the magnetic force does positive (amplification) or negative (damping) work on the CNT resonator during one oscillation period. The answer turns out to depend on the "sign" of the extra spin, which in its turn depends on which of the two oppositely magnetized electrodes is the source of spin-polarized electrons injected into the CNT-QD. But this can be changed by changing the polarity of the voltage bias applied between the two ferromagnetic electrodes. Hence we can be certain that for either positive or negative polarity, energy will be pumped into the vibrations of the CNT resonator giving rise to a mechanical instability and to pronounced vibrations if the pumping can overcome the dissipation in the mechanical subsystem.

In order to rigorously demonstrate the phenomenon that we so far have only described qualitatively for our model system, we assume that the dynamics of the CNT-QD resonator is completely characterized by the amplitude of its fundamental bending mode $u(t)$, whose time evolution is governed by Newton's equation for a harmonic oscillator of eigenfrequency $\omega_{0}$,

$$
m\left[\ddot{u}+\gamma \dot{u}+\omega_{0}^{2} u\right]=F \operatorname{Tr}\left\{\hat{\rho}\left(\hat{n}_{\downarrow}-\hat{n}_{\uparrow}\right)\right\}+F_{\text {elast }}^{\mathrm{eq}} .
$$

Here $\gamma=\omega_{0} / Q_{0}$ is a phenomenological damping rate, $Q_{0}$ is the mechanical quality factor, and $m$ is the effective mass of the resonator. The first force term on the rhs, where $F=\left(g \mu_{B} / 2\right) \partial B_{z}(u) /\left.\partial u\right|_{u=0}$ and $\hat{n}_{\sigma}=d_{\sigma}^{\dagger} d_{\sigma}$ is the density operator for QD electrons with spin $\sigma=\uparrow, \downarrow$, is the magnetic force induced by the interaction between the QD spin and the gate-induced inhomogeneous magnetic field $B_{z}(u)$, which is assumed to have a linear dependence on $u$ in the region of interest. The second force term is the elastic restoring force that compensates the magnetic force in the absence of microwave irradiation introduced so that the deflection coordinate $u$ (along the $z$ axis, see Fig. 1) is measured from the midpoint of the (bent) resonator in this static case. The trace in Eq. (1) is over the electronic degrees of freedom. Consequently, $\hat{\rho}$ is the electron density matrix operator, which obeys the quantum Liouville-von Neumann equation $(\hbar=1)$

$$
i \frac{d \hat{\rho}}{d t}=\left[\hat{H}_{d}+\hat{H}_{l}+\hat{H}_{t}, \hat{\rho}\right] .
$$

Here $\hat{H}_{d}$ is the QD Hamiltonian,

$$
\begin{aligned}
\hat{H}_{d}= & \varepsilon_{0}\left(\hat{n}_{\uparrow}+\hat{n}_{\downarrow}\right)+\frac{g \mu_{B} B_{z}(u)}{2}\left(\hat{n}_{\uparrow}-\hat{n}_{\downarrow}\right) \\
& +\frac{g \mu_{B} h}{2} \cos (\Omega t)\left(d_{\uparrow}^{\dagger} d_{\downarrow}+d_{\downarrow}^{\dagger} d_{\uparrow}\right)-U d_{\uparrow}^{\dagger} d_{\downarrow}^{\dagger} d_{\uparrow} d_{\downarrow},
\end{aligned}
$$

where $\varepsilon_{0}$ is the energy of the QD level relative to the Fermi energy in the leads, $g \mu_{B} B_{z}(u)$ is the CNT's deflectiondependent Zeeman energy splitting, $h$ is the amplitude of the magnetic component of the external microwave field, which oscillates with frequency $\Omega$ along the $x$ axis [see Fig. 1(a)], and $U$ is the Coulomb (charging) energy cost for double occupancy of the QD. Assuming that the variation of the magnetic field $B_{z}$ along the mechanical motion is small compared to its value at the equilibrium position of the dot, $\Delta=g \mu_{B} B_{z}(u=0)$, we retain only the linear term in its Taylor expansion, $g \mu_{B} B_{z}(u)=\Delta+2 F u$.

The second term in the commutator in Eq. (2) describes noninteracting electrons in the electrodes (for now assumed to be $100 \%$ spin polarized "half metals" [9]) while the third term,

$\hat{H}_{t}=\sum_{k} \mathcal{T}\left[e^{i e V t / 2} c_{L k \uparrow}^{\dagger} d_{\uparrow}+e^{-i e V t / 2} c_{R k \downarrow}^{\dagger} d_{\downarrow}\right]+$ H.c.,

is a standard tunnel Hamiltonian. Here electron tunneling is characterized by the overlap integral $\mathcal{T}$, a bias voltage $V$ is applied between the electrodes, and $c_{j k \sigma}^{\dagger}$ are electron creation operators acting in the left $(j=L)$ and right $(j=R)$ electrodes. For simplicity, we take the amplitudes for tunneling to the left and right to be the same [10]. The magnetization in the source (left if $V>0$ ) electrode and in the magnetic tip are assumed to be antiparallel to each other [11]. To investigate the parallel case, one has to reverse the polarity of the bias voltage, $V \rightarrow-V$, in Eq. (4), so that the right electrode becomes the source.

By treating the tunnel Hamiltonian perturbatively and using the reduced density matrix approach $[12,13]$, one can factorize the density operator, $\hat{\rho}(t)=\hat{\varrho}_{d}(t) \otimes \hat{\rho}_{l}$, into a product of an equilibrium density operator for the leads at temperature $T, \hat{\rho}_{l}=\exp \left(-\hat{H}_{l} / k_{B} T\right)$, and a reduced density operator for the QD states,

$$
\hat{\varrho}_{d}=c_{0}|0\rangle\left\langle 0\left|+c_{2}\right| \uparrow \downarrow\right\rangle\langle\uparrow \downarrow|+| \sigma\rangle \varrho_{\sigma \sigma^{\prime}}\left\langle\sigma^{\prime}\right| .
$$

In Eq. (5), $|\sigma\rangle=d_{\sigma}^{\dagger}|0\rangle,|\uparrow \downarrow\rangle=d_{\uparrow}^{\dagger} d_{\downarrow}^{\dagger}|0\rangle$ are singly and doubly occupied electron states, $\varrho_{\sigma \sigma^{\prime}}=\left\langle\sigma\left|\hat{\varrho}_{d}\right| \sigma^{\prime}\right\rangle$ and $c_{0}, c_{2}$ are normalization constants. We consider a NEM system in the strong Coulomb blockade regime, $U \gg\left|e V / 2 \pm \varepsilon_{0}\right|$, for which double electron occupancy of the dot is forbidden, so that $c_{2}=0$. By averaging over the electronic states in the leads, we get an equation for the matrix 


$$
\hat{\varrho}=\left(\begin{array}{cc}
\varrho_{\uparrow \uparrow} & \varrho_{\uparrow \downarrow} \\
\varrho_{\downarrow \uparrow} & \varrho_{\downarrow \downarrow}
\end{array}\right)
$$

which characterizes the state of the singly occupied QD level. This equation reads

$\dot{\hat{\varrho}}=-\frac{i}{2}\left[(\Delta+2 F u) \sigma_{z}+\nu_{h} \cos (\Omega t) \sigma_{x}, \hat{\varrho}\right]-\frac{\Gamma}{2} \mathfrak{L}[\hat{\varrho}]$,

where

$$
\mathfrak{L}[\hat{\varrho}]=\frac{3}{2} \hat{\varrho}+\frac{1}{2} \sigma_{z} \hat{\varrho} \sigma_{z}+2 \sigma_{\kappa} \hat{\varrho} \sigma_{-\kappa}-\left(\hat{1}+\kappa \sigma_{z}\right) .
$$

Here $\sigma_{x, y, z}$ are the Pauli matrices, $\sigma_{ \pm}=\left(\sigma_{x} \pm i \sigma_{y}\right) / 2$, $\nu_{h}=g \mu_{B} h, \Gamma=2 \pi \nu|\mathcal{T}|^{2}$ is the energy level width, and $\nu$ is the density of states in the leads. In Eq. (8), $\kappa=\operatorname{sign}(e V)$ determines the relative magnetization in the source and the tip: $\kappa=+1$ stands for antiparallel, while $\kappa=-1$ stands for parallel alignment. The first term in Eq. (7) describes the dynamical part of the time evolution of the electronic subsystem, while the second term is a stochastic part associated with tunneling processes from/to the leads. We assume the condition of a large bias voltage, $|e V| \gg\left(k_{B} T, \Delta, \Omega, \Gamma\right)$.

Having in mind microwave-field intensities that can be achieved experimentally in the $\mathrm{THz}$ frequency range, we will consider a situation where $\nu_{h} \ll \Omega \sim \Delta$. Under this condition, one can apply the rotating wave approximation, which makes it possible to remove the explicit time dependence in Eq. (7) by a unitary transformation. As a result, we may replace $\nu_{h} \cos (\Omega t)$ by $\nu_{h} / 2$ and renormalize $(\Delta+2 F u) \rightarrow(\Delta-\Omega+2 F u)$ in Eq. (7). We then evaluate Eq. (7) under the assumption that the motion of the QD is adiabatic $\left(\omega_{0} \ll \Gamma\right)$, so that the characteristic times associated with mechanical motion and electron tunneling are well separated. It follows that the equation of motion (1) for a small-amplitude microwave field, $\nu_{h} \lesssim \Gamma$, takes the form

$$
\ddot{u}+\gamma \dot{u}+\omega_{0}^{2} u=w_{1}^{2} f_{1}(u)+\frac{w_{2}^{2}}{\Gamma} f_{2}(u) \dot{u},
$$

where (as before) $u$ is the CNT's deflection relative to its equilibrium position in the absence of a microwave field,

$$
w_{1}^{2}=\frac{3 F \nu_{h}^{2}}{4 m \Gamma^{2}}, \quad w_{2}^{2}=\frac{4 F^{2} \nu_{h}^{2}}{m \Gamma^{3}},
$$

and

$f_{1}(u)=\frac{\kappa}{1+4 J^{2}(u)}, \quad f_{2}(u)=\kappa J(u) \frac{4 J^{2}(u)+13}{\left[1+4 J^{2}(u)\right]^{3}}$,

where $J(u)=(\Delta-\Omega+2 F u) / \Gamma$. The function $f_{1}(u)$ describes a nonlinear force, while the function $f_{2}(u)$ is a nonlinear friction term. Both functions depend on the spin accumulated on the QD due to photo-induced electronic spin-flip transitions; hence, their values vary rapidly as the deflection $u$ of the CNT-QD resonator is close to satisfying the resonance condition $J\left(u=u_{r}\right)=0$, where the rate of spin-flip transitions is maximal.

Whether or not microwave induced spin-flip transitions will contribute a "negative friction" $\left(f_{2}>0\right)$, possibly leading to a nanomechanical instability, depends, as will be shown by the stability analysis that follows, on whether resonant spin-flip transitions occur for a deflection towards $\left(u_{r}>0\right)$ or away from $\left(u_{r}<0\right)$ the magnetic gate.

Linearizing Eq. (9) assuming $|u| \ll \operatorname{Max}\left\{\left|u_{r}\right|, \Gamma / F\right\}$ corresponds to expanding $f_{1}(u)$ to first and $f_{2}(u)$ to zeroth order in $u$. Neglecting the former terms, which lead to a small renormalization of the static shift and vibration frequency of the resonator, we obtain an expression for the imaginary part of the eigenfrequency,

$$
\operatorname{Im}[\omega]=\left(\gamma-\gamma_{h}\right) / 2,
$$

where $\gamma_{h} \propto f_{2}(0)$ and

$$
\gamma_{h}=\kappa(\Delta-\Omega) w_{2}^{2} \Gamma^{2} \frac{4(\Delta-\Omega)^{2}+13 \Gamma^{2}}{\left[4(\Delta-\Omega)^{2}+\Gamma^{2}\right]^{3}}
$$

is the microwave-induced "friction" coefficient. We note that the amplitude of the mechanical oscillations increases with time if $\gamma_{h}>\gamma$, corresponding to a mechanical "shuttling" instability. To determine the instability criterion, we neglect the intrinsic friction $(\gamma=0)$ of the mechanical subsystem. Then, for antiparallel magnetization between tip and source $(\kappa=+1)$, shuttling occurs when the microwave frequency is lower than the Zeeman energy splitting, $\Omega<\Delta$. Therefore, whether an instability occurs or not depends on whether the deflection $u_{r}=$ $(\Omega-\Delta) / 2 F$ that corresponds to resonant spin-flip transitions is in the direction of the magnetic gate $\left(u_{r}>0\right)$ or away from it $\left(u_{r}<0\right)$. The latter case gives rise to an instability, while the former leads to additional damping and stability. The corresponding criterion for parallel magnetizations $(\kappa=-1)$ can be found from Eq. (13) in a similar manner.

The instability threshold for CNT oscillations is conditioned by the equality of the microwave field-dependent contribution to the quality factor, $Q_{h}=\omega_{0} / \gamma_{h}$, and the intrinsic mechanical quality factor $Q_{0}$. This criterion determines the critical value for the amplitude of the microwave field. Estimating $Q_{h}$ by using the maximum value of the increment $(|\Delta-\Omega| \sim \Gamma)$ given by Eq. (13), we find that

$$
Q_{h}^{-1} \sim \frac{1}{\omega_{0}} \frac{w_{2}^{2}}{\Gamma} \sim u_{0}^{2} \frac{F^{2}}{\Gamma^{2}} \frac{\nu_{h}^{2}}{\Gamma^{2}}
$$


where $u_{0}=\left(2 m \omega_{0}\right)^{-1 / 2} \simeq 2 \mathrm{pm}$ (see, e.g., [14]) is the amplitude of the CNT's zero-point oscillation. Using the realistic values $1 \mathrm{~T}$ for the magnetic field $B_{z}(0)$ and $5 \times 10^{6} \mathrm{~T} / \mathrm{m}$ for the field gradient $\partial B_{z}(0) / \partial z[15,16]$, while taking the CNT quality factor $Q_{0}$ and the tunnel coupling $\Gamma$ to be $10^{6}$ [17] and $1 \times 10^{9} \mathrm{~s}^{-1}$ [10], respectively, one then finds a lower bound of $h \sim 1 \mathrm{mT}$ for the magnetic amplitude of the microwave field.

A partial rather than complete spin polarization of the leads would not qualitatively change the threshold for shuttling since it only produces quantitative changes in the leads' densities of state and modifies $\Gamma_{L(R)}^{\uparrow, \downarrow}$. As a result, direct electron transfer processes between the left and right electrodes without spin-flips become possible, hence reducing the spin-flip rate. The magnitude of the field induced friction is proportional to the degree of spin polarization $\eta=\left|\Gamma^{\uparrow}-\Gamma^{\downarrow}\right| /\left(\Gamma^{\uparrow}+\Gamma^{\downarrow}\right)$, where $\Gamma^{\uparrow(\downarrow)}=\Gamma_{L}^{\uparrow(\downarrow)}=\Gamma_{R}^{\downarrow(\uparrow)}$. Therefore, for partially polarized leads $(\eta<1)$ the rate at which the oscillation amplitude grows if the shuttle instability criterion is met decreases with $\eta$ as $\gamma_{h} \propto \eta(1+\eta)$ and vanishes in the limit $\eta \rightarrow 0$.

If there is a shuttle instability the amplitude of the resonator vibrations increases with time and we have to consider the full nonlinear version of the equation of motion (9). In this case, we use the Krylov-Bogoliubov (KB) method [18] for our analysis. The KB ansatz is based on the assumption that the CNT displacement takes the form $u(t)=A(t) \sin \left(\omega_{0} t+\psi(t)\right)$, where both the amplitude $A(t)$ and the phase $\psi(t)$ are slowly varying functions of time and the CNT mechanical energy is $m \omega_{0}^{2} A^{2} / 2$. Substituting into Eq. (9), multiplying by $\dot{u}(t)$, and averaging over one oscillation period, we get an equation for the rate of change of $A(t)$,

$$
\begin{aligned}
\frac{d A^{2}}{d t} & =\omega_{0}\left(W_{1}(A)-W_{0}(A)\right), \\
W_{1}(A) & =-\frac{A^{2}}{\pi}\left(u_{0} \frac{2 F \nu_{h}}{\Gamma^{2}}\right)^{2} \int_{0}^{2 \pi} \cos ^{2}(\psi) f_{2}[A \sin (\psi)] d \psi,
\end{aligned}
$$

where $W_{1}(A)$ is proportional to the work done on the NEM system by the magnetic force during one oscillation period and $W_{0}(A)=A^{2} / Q_{0}$ is proportional to the energy dissipated due to intrinsic friction during the same time; the solution $W_{1}(A)=W_{0}(A)$ yielding a stationary solution. In Fig. 2, the ratio $W_{1}(A) / W_{0}(A)$ is plotted as a function of $A$ for three different microwave intensities $\left(\nu_{h}\right)$, corresponding to three different regimes. Regime (i) occurs at low microwave intensities when the work done by the magnetic force is smaller than the dissipated energy $\left(W_{1} / W_{0}<1\right)$ for any oscillation amplitudes and hence the only stationary solution is static, $A=0$. Regime (ii) arises at intermediate radiation intensities where

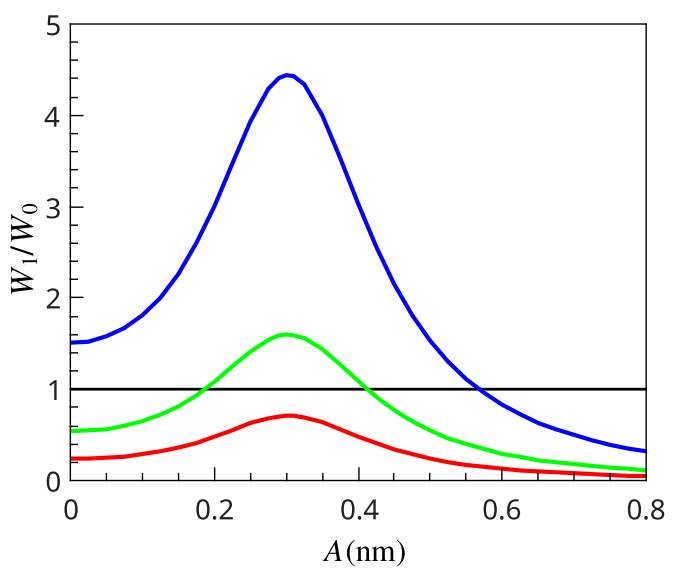

FIG. 2. Ratio between work done on the NEM system by the magnetic force $\left(\propto W_{1}\right)$ and energy dissipated due to intrinsic friction $\left(\propto W_{0}\right)$ plotted as a function of CNT oscillation amplitude $A$ for $\Gamma=0.6 \times 10^{9} \mathrm{~s}^{-1}, \Delta-\Omega=\Gamma, u_{0}=2 \mathrm{pm}$, $\partial B_{z} / \partial z=5 \times 10^{6} \mathrm{~T} / \mathrm{m}, Q_{0}=10^{6}$, and three different microwave field intensities $\nu_{h}$. If $W_{1} / W_{0}<1$ for some $A$, then $A$ decreases with time according to Eq. (15), while if $W_{1} / W_{0}>1$, $A$ increases with time. If $W_{1} / W_{0}=1$ (black horizontal help line) for some finite amplitude, then Eq. (15) has a nontrivial $(A \neq 0)$ stationary solution for that amplitude. The red (bottom) curve $\left(\nu_{h}=0.4 \Gamma\right)$ describes the behavior of the NEM system below the instability threshold and illustrates the text's regime (i), which has no nontrivial stationary solution. The green (middle) curve $\left(\nu_{h}=0.6 \Gamma\right)$ corresponds to regime (ii), where the mechanical system is characterized by two nontrivial stationary solutions. The blue (top) curve $\left(\nu_{h}=1.0 \Gamma\right)$ corresponds to regime (iii), with a single nontrivial stationary solution to Eq. (15) for $A=A_{\text {lim }}$. In this regime, a mechanical instability leads to self-sustained resonator oscillations of amplitude $A_{\lim }$ [20].

$d A^{2} / d t=0$ for three different amplitudes, each corresponding to a stationary solution. Two of these solutions, one static and one with finite oscillation amplitude, are stable while the third (finite amplitude) solution is unstable. Note that if the NEM system is initially static, it will remain so in this regime. The third regime (iii) emerges at high intensities of the microwave radiation where the intrinsic mechanical dissipation overcomes the work performed by magnetic forces only at a certain finite mechanical vibration amplitude $A_{\text {lim }}$, which defines the amplitude of the CNT self-sustained oscillations. Together, the intensity regimes (i)-(iii) determine a so-called hard instability scenario [19], which results in a hysteretic behavior of the system characteristics as a function of the microwave field intensity $\nu_{h}$.

The self-sustained oscillations, being due to electronic spin-flip transitions, will generate a correction $\delta I \propto A_{\lim }^{2}$ to the electrical current through the NEM system, which can be detected. An estimation using the system parameters given in Fig. 2 shows that $|\delta I| \sim 10-100$ pA. Alternatively, the CNT bending vibrations can be detected by using 
standard methods such as the rectification technique of Ref. [14] or the scanning force microscopy technique of Ref. [21].

In conclusion, we have suggested a new spin-mediated photomechanical mechanism for coupling electronic and mechanical degrees of freedom in a nanoelectromechanical (NEM) device comprising a carbon nanotube (CNT) resonator suspended between two magnetic electrodes and under the influence of an inhomogeneous magnetic field from a nearby tip-shaped magnetic gate. The predicted strong effect of microwave radiation on the nanomechanics of the device is based on two features: (i) the resonant nature of the microwave-induced electronic spin-flip transitions in the CNT resonator, and (ii) a latent nanomechanical instability of the resonator caused by a spin-dependent magnetic force that pumps energy into its vibrations. The instability occurs if the pumping rate [see Eq. (14)] exceeds the dissipation rate, set by the quality factor $Q_{0}$ of the resonator. We find that this criterion leads to a lower bound of about $1 \mathrm{mT}$ for the amplitude of the magnetic component $(h)$ of the microwave field.

The predicted photo-induced mechanical instability develops into pronounced vibrations of the CNT resonator. These are accompanied by temporal oscillations of the spin accumulated in the CNT and related significant effects on the spin-dependent electrical current through the device corresponding to a highly efficient (up to 100\%) photoelectric transduction effect. The results obtained for the studied CNT-based magnetic NEM device provide a basis for pursuing further spintronics applications, including but not limited to spin-current rectifiers (filters, splitters, ratchets, etc.), and as elements of ac and dc circuits and devices with controllable (nonlinear) damping.

We acknowledge fruitful discussions with A. Hüttel and S. Ludwig. This work was partially supported by the Swedish Research Council (VR).

[1] K. L. Ekinci and M. L. Roukes, Rev. Sci. Instrum. 76, 061101 (2005).

[2] R. I. Shekhter, L. Y. Gorelik, I. V. Krive, S. I. Kulinich, M. N. Kiselev, A. V. Parafilo, K. Kikoin, and M. Jonson, Fiz. Nizk. Temp. 40, 775 (2014) [Low Temp. Phys. 40, 600 (2014)].
[3] M. Poot and H. S. J. van der Zant, Phys. Rep. 511, 273 (2012).

[4] K. Flensberg and C. M. Marcus, Phys. Rev. B 81, 195418 (2010).

[5] P. Stadler, W. Belzig, and G. Rastelli, Phys. Rev. Lett. 113, 047201 (2014).

[6] R. I. Shekhter, O. Entin-Wohlman, and A. Aharony, Phys. Rev. Lett. 111, 176602 (2013).

[7] R. I. Shekhter, A. Pulkin, and M. Jonson, Phys. Rev. B 86, 100404 (2012)

[8] J. Atalaya and L. Y. Gorelik, Phys. Rev. B 85, 245309 (2012).

[9] M. Ziese, Rep. Prog. Phys. 65, 143 (2002).

[10] For asymmetric tunnel couplings and optimal resonance conditions, $|\Omega-\Delta| \sim \Gamma_{D}, \Gamma$ in Eq. (14) should be replaced by $\Gamma_{D}\left(\Gamma_{D} \ll \Gamma_{S}\right)$ or $\sqrt{\Gamma_{S} \Gamma_{D}}\left(\Gamma_{D} \gg \Gamma_{S}\right)$. This will not qualitatively change the NEM instability criterion.

[11] Note that the direction of the magnetic moment of an electron is opposite to that of its spin.

[12] T. Novotny, A. Donarini, and A.-P. Jauho, Phys. Rev. Lett. 90, 256801 (2003).

[13] D. Fedorets, L. Y. Gorelik, R. I. Shekhter, and M. Jonson, Phys. Rev. Lett. 95, 057203 (2005); New J. Phys. 7, 242 (2005).

[14] A. K. Hüttel, G. A. Steele, B. Witkamp, M. Poot, L. P. Kouwenhoven, and H. S. J. van der Zant, Nano Lett. 9, 2547 (2009).

[15] C. L. Degen, M. Poggio, H. J. Mamin, C. T. Rettner, and D. Rugar, Proc. Natl. Acad. Sci. U.S.A. 106, 1313 (2009).

[16] F. Xue, P. Peddibhotla, M. Montinaro, D. P. Weber, and M. Poggio, Appl. Phys. Lett. 98, 163103 (2011).

[17] J. Moser, A. Eichler, J. Güttinger, M. I. Dykman, and A. Bachtold, Nat. Nanotechnol. 9, 1007 (2014).

[18] N. Bogolubov, Asymptotic Methods in the Theory of Non-Linear Oscillations (Gordon and Breach, Paris, 1961).

[19] A. Isacsson, L. Y. Gorelik, M. V. Voinova, B. Kasemo, R. I. Shekhter, and M. Jonson, Physica (Amsterdam) 255B, 150 (1998).

[20] Typical values of the amplitudes of the developed mechanical vibrations are set by the distance between the point defined by the static solution: $A=0$ and emergent point where the spin flip assisted resonant absorption of the microwave field is achieved.

[21] D. Garcia-Sanchez, A. San Paulo, M. J. Esplandiu, F. Perez-Murano, L. Forró, A. Aguasca, and A. Bachtold, Phys. Rev. Lett. 99, 085501 (2007). 